\title{
Effect of lateral tip stiffness on atomic-resolution force field spectroscopy
}

Berkin Uluutku, and Mehmet Z. Baykara

Citation: Journal of Vacuum Science \& Technology B, Nanotechnology and Microelectronics: Materials, Processing, Measurement, and Phenomena 31, 041801 (2013); doi: 10.1116/1.4807376

View online: http://dx.doi.org/10.1116/1.4807376

View Table of Contents: http://avs.scitation.org/toc/jvb/31/4

Published by the American Vacuum Society

\section{Articles you may be interested in}

Artifacts related to tip asymmetry in high-resolution atomic force microscopy and scanning tunneling microscopy measurements of graphitic surfaces

Journal of Vacuum Science \& Technology B, Nanotechnology and Microelectronics: Materials, Processing, Measurement, and Phenomena 33, 031802 (2015); 10.1116/1.4915898

Three-dimensional interaction force and tunneling current spectroscopy of point defects on rutile $\mathrm{TiO}_{2}(110)$ Applied Physics Letters 108, 071601 (2016); 10.1063/1.4942100

Tribological interaction between polytetrafluoroethylene and silicon oxide surfaces The Journal of Chemical Physics 141, 164702 (2014); 10.1063/1.4898384

Combined low-temperature scanning tunneling/atomic force microscope for atomic resolution imaging and sitespecific force spectroscopy

Review of Scientific Instruments 79, 033704 (2008); 10.1063/1.2842631

Image contrast mechanisms in dynamic friction force microscopy: Antimony particles on graphite Journal of Applied Physics 121, 044307 (2017); 10.1063/1.4974882

Low noise current preamplifier for qPlus sensor deflection signal detection in atomic force microscopy at room and low temperatures

Review of Scientific Instruments 88, 073702 (2017); 10.1063/1.4993737 \\ Instruments for Advanced Science}

Contact Hiden Analytical for further details: W www.HidenAnalytical.com E info@hiden.co.uk CLICK TO VIEW our product catalogue

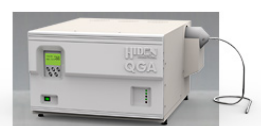

Gas Analysis

dynamic measurement of reaction gas streams , catalysis and thermal analysis molecular beam studies , molecular beam studies 'dissolved species probes

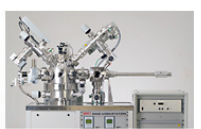

Surface Science

UHVTPD

end point detection in ion beam etch elemental imaging - surface mapping

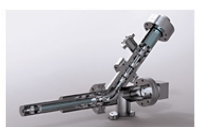

Plasma Diagnostics plasma source characterization etch and deposition process reaction kinetic studies analysis of neutral and radical species

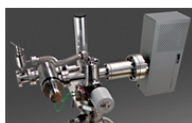

Vacuum Analysis partial pressure measurement and control of process gases reactive sputter process control vacuum diagnostics vacuum coating process monitoring 


\title{
Effect of lateral tip stiffness on atomic-resolution force field spectroscopy
}

\author{
Berkin Uluutku \\ Department of Mechanical Engineering, Bilkent University, Ankara 06800, Turkey \\ Mehmet Z. Baykara ${ }^{a)}$ \\ Department of Mechanical Engineering, Bilkent University, Ankara 06800, Turkey \\ and UNAM-Institute of Materials Science and Nanotechnology, Bilkent University, Ankara 06800, Turkey
}

(Received 6 March 2013; accepted 6 May 2013; published 20 May 2013)

\begin{abstract}
Atomic force microscopy is being increasingly used to measure atomic-resolution force fields on sample surfaces, making correct interpretation of resulting data critically important. In addition to asymmetry, elastic deformations undergone by the microscope tip are thought to affect measurements. In this study, simple analytical potentials and a model tip apex were used to theoretically analyze how lateral tip stiffness affects force spectroscopy on the surface of $\mathrm{NaCl}(001)$. The results suggest that lateral deformations experienced by the tip lead to certain distortions in measured force spectra, the degree of which depends on lateral tip stiffness. (c) 2013 American Vacuum Society. [http://dx.doi.org/10.1116/1.4807376]
\end{abstract}

\section{INTRODUCTION}

Among the large family of scanning probe microscopy techniques, noncontact atomic force microscopy (NC-AFM) has special significance due to its ability to image both conducting and insulating surfaces with atomic resolution, and also its usefulness in recording tip-sample force interactions with atomic precision, resulting in atom-specific force mapping with $\mathrm{pm}$ and $\mathrm{pN}$ resolution. ${ }^{1-3}$ In the last few years, the force spectroscopy capability of NC-AFM has been extended to two and three spatial dimensions, and multiple research groups are now able to routinely record 2D and 3D maps of interaction forces on various sample surfaces with atomic resolution (2D/3D-AFM). ${ }^{4}$ As is the case with any experimental approach that has not yet fully matured, results' interpretation may become controversial, especially when the physical mechanisms responsible for distortions and artifacts in experimental data are not fully understood. Accordingly, both experimental and theoretical investigations continue to be performed by the NC-AFM community in order to (i) improve the reproducibility of 2D/3D-AFM experiments and (ii) better understand the physical reasons as well as the characteristic signatures of certain artifacts frequently encountered during data acquisition. ${ }^{5-7}$ In this article, we take a further step toward these goals by theoretically investigating the influence of lateral tip stiffness on atomic-resolution force spectroscopy measurements. Calculations were performed with simple analytical potentials using a model tip apex on the sample surface of $\mathrm{NaCl}(001)$. Rather than focusing on atomic-scale displacements at the very end of the tip apex ${ }^{8}$ or normal-force induced bending of asymmetric tip apices in a preferred direction, ${ }^{5,9}$ our present approach investigates the influence of elastic deformations undergone by a symmetric tip with a rigid apex due to local lateral forces on atomic-resolution force spectroscopy.

Like other AFM techniques, the basic operational principle of NC-AFM relies on measuring the changes in certain

${ }^{\text {a)} E l e c t r o n i c ~ m a i l: ~ m e h m e t . b a y k a r a @ b i l k e n t . e d u . t r ~}$ experimental parameters caused by the interactions of a sharp probe tip with a sample surface of interest. In the case of NC-AFM, the sharp (often idealized as terminating in a single atom) probe tip is usually attached to a micromachined cantilever oscillating at resonance. Changes in resonance frequency caused by tip-sample interactions at small tip-sample distances (often $<1 \mathrm{~nm}$ ) are detected during both imaging and spectroscopy experiments. ${ }^{10}$ As expected for experiments performed at the nanometer length scale, phenomena such as thermal drift and variabilities in tip structure, asymmetry, and chemistry significantly affect the acquired data and cause difficulties in achieving reproducibility in experiments performed with different probe tips. ${ }^{5}$ Additionally, lateral relaxations experienced by the probe tip due to interaction forces are thought to lead to distortions in atomic-resolution force spectroscopy maps..$^{6,7,11}$ The degree of these distortions would thus ultimately depend on the lateral stiffness of the probe tips employed in the experiments, as well as tip-sample distance. It is clearly important to form a basic understanding regarding the relationship between tip stiffness and related effects on force spectroscopy using a simple, model tip-sample system and analytical potentials.

\section{MODELING}

The results presented here have been obtained using a model tip apex consisting of three $\mathrm{Pt}$ atoms in a closepacked, planar configuration and a sample $\mathrm{NaCl}(001)$ surface comprising $50 \times 50$ ions (Fig. 1). MATLAB ${ }^{12}$ has been used to compute the vertical and lateral force interactions between tip apex atoms and those on the sample surface using well-known Lennard-Jones (L-J) and ionic interaction models and Lorentz-Berthelot mixing rules. ${ }^{5,13}$ Thereby, at every point of a 1-pm-step mesh above the surface, the interaction force components between each tip apex atom and surface atom have been summed up to calculate the total interaction force acting on the model tip apex. Since binary ionic crystals are frequently used as samples for NC-AFM experiments $^{6-8,14-17}$ (mostly due to their ease of preparation through cleaving, as well as attractive features such as 


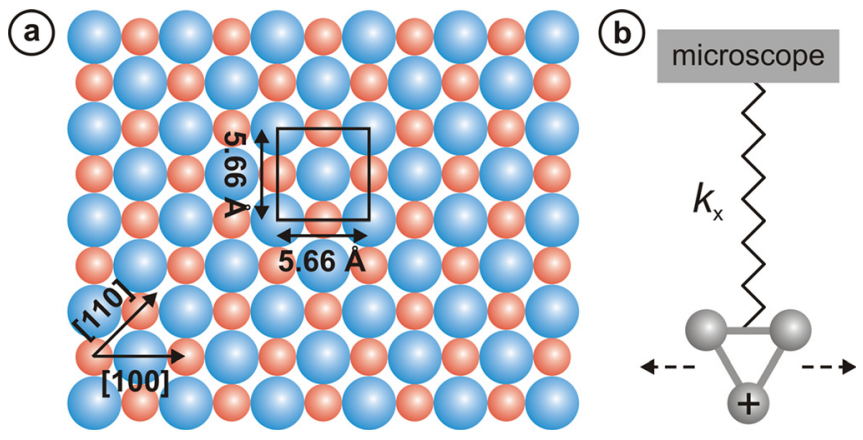

FIG. 1. (Color online) (a) Structural model of the $\mathrm{NaCl}(001)$ surface, together with a unit cell and crystallographic directions of interest. Large, blue spheres represent $\mathrm{Cl}^{-}$ions, whereas small, red spheres represent $\mathrm{Na}^{+}$ions. (b) Simple model of a tip apex consisting of three Pt atoms connected to the microscope base by an elastic spring of lateral stiffness $k_{\mathrm{x}}$, allowing lateral deflections of the apex under the influence of interaction forces. Only the frontmost Pt atom is charged. Please note that vertical deflections are not currently considered in our analysis as experimentally determined vertical stiffness values are generally found to be significantly larger than their lateral counterparts in atomic-scale AFM experiments. ${ }^{22}$

forming large flat terraces and serving as model substrates for molecular electronic devices), the choice of $\mathrm{NaCl}(001)$ as a model surface is justified. It should be noted that only the frontmost Pt atom in the tip apex is assumed to be charged (with a positive unity charge), as this has been found to be a reasonable assumption in previous studies. ${ }^{5,18}$ Being inspired by models used in pioneering theoretical studies of stick-slip in friction force microscopy, the three-atom model tip apex used in our investigations is assumed to be attached to the microscope base by an elastic spring with a certain effective lateral stiffness value $k_{\mathrm{x}}$ [Fig. 1(b)]. ${ }^{19,20}$ It is important to indicate that the effective $k_{\mathrm{x}}$ value mentioned here not only comprises the elastic behavior of the cantilever to which the tip is attached (through its torsional spring constant) but also the lateral spring constant of the tip itself, which is often much lower, and thus largely determines the effective lateral spring constant value $k_{\mathrm{x}}$, with most reported values in the literature between 5 and $25 \mathrm{~N} / \mathrm{m} .{ }^{20-22}$ Lastly, it should be indicated that in an actual force mapping experiment performed via NC-AFM, the calculated interactions will be averaged over the oscillation cycle of the cantilever; however, the detected frequency shifts will still be largely dominated by interactions in the small tip-sample distances covered in the present discussion.

\section{RESULTS AND DISCUSSION}

Before switching to an analysis of the effect of lateral tip stiffness on atomic-resolution force spectroscopy, it would be useful to study the interaction forces experienced by the model tip described in Sec. II, under ideal stiffness conditions $\left(k_{\mathrm{x}}=\infty\right)$. Figure 2 displays two-dimensional interaction maps of vertical and lateral forces $\left(F_{\mathrm{z}}\right.$ and $F_{\mathrm{x}}$, respectively) along two crystallographic directions: [100] and [110]. While the [110] direction includes only one type of ion $\left(\mathrm{Na}^{+}\right.$for the specific cut described here), the [100] direction includes both $\mathrm{Na}^{+}$and $\mathrm{Cl}^{-}$ions. The interaction maps, calculated with lateral and vertical step sizes of $\sim 1.5 \mathrm{pm}$ and tip-sample distances of $3-6 \AA$, reveal, as expected, that the positively charged tip apex experiences the largest attractive vertical forces on top of $\mathrm{Cl}^{-}$ions, while the largest repulsive vertical forces are detected on $\mathrm{Na}^{+}$ions for both crystallographic directions [Figs. 2(a) and 2(c)]. Ionic interactions dominate L-J for a majority of simulated heights, quantitatively in line with previous findings on ionic crystal surfaces. ${ }^{5}$ Simulated lateral forces exhibit a more
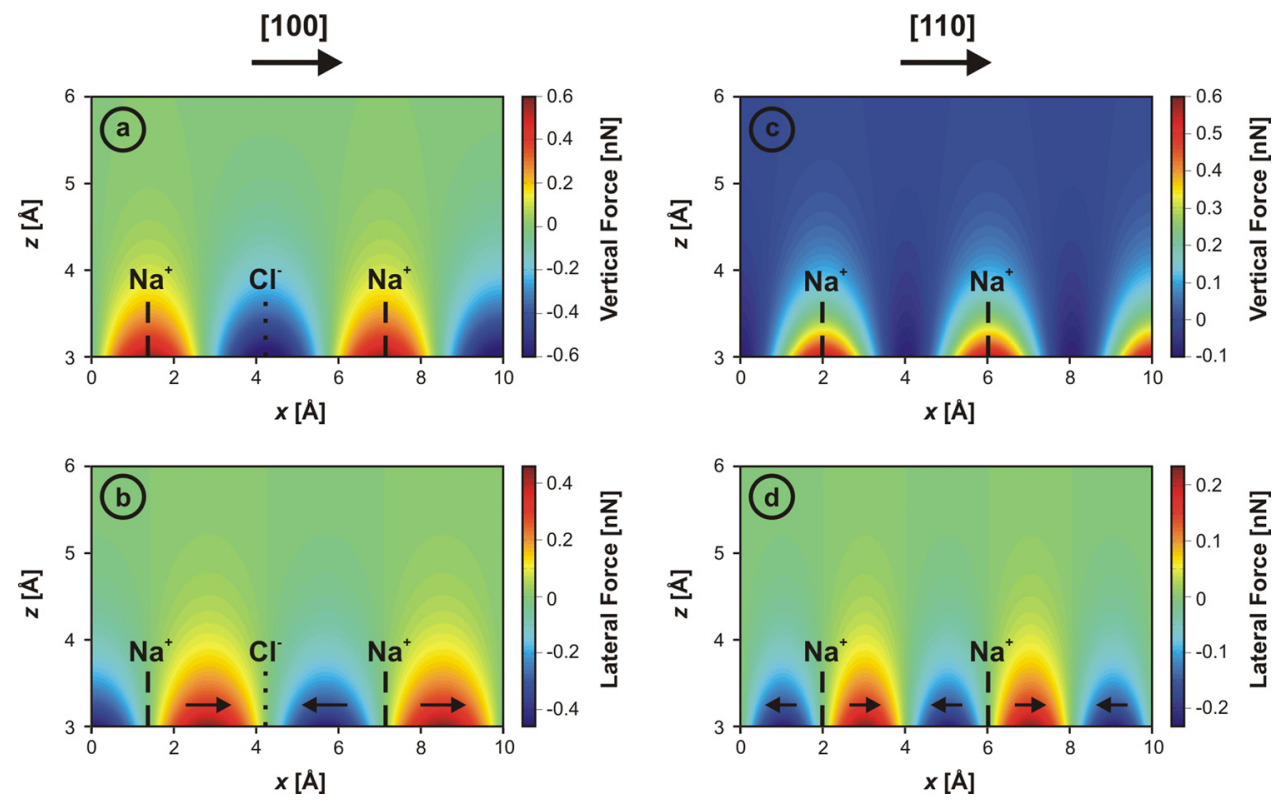

FIG. 2. (Color online) 2D maps of vertical and lateral interaction forces experienced by the model tip apex along [100] [(a) and (b)] and [110] [(c) and (d)] directions under ideal $\left(k_{\mathrm{x}}=\infty\right)$ stiffness conditions. The positions of $\mathrm{Na}^{+}$and $\mathrm{Cl}^{-}$ions are labeled and indicated with dashed and dotted lines, respectively. For vertical force maps [(a) and (c)] positive forces are repulsive, and negative forces are attractive. For lateral force maps [(b) and (d)], positive forces point to the right and negative forces point to the left, as indicated with arrows. Please note that the color scales have been approximately set to the maximum and minimum force values observed in each map to better reflect the differences between atomic sites. 


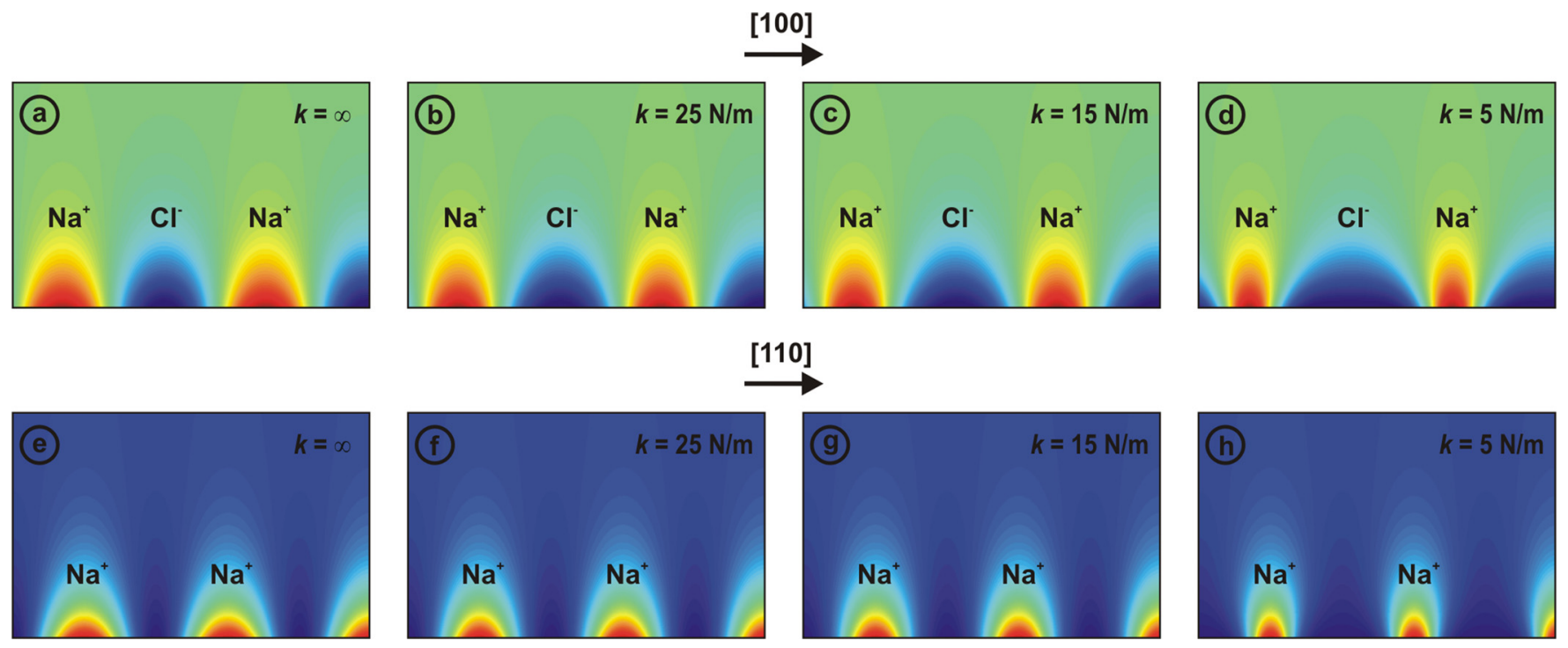

FIG. 3. (Color online) 2D maps of vertical interaction forces experienced by the model tip apex along [100] and [110] directions with lateral tip stiffness ( $k_{\mathrm{x}}$ ) values of $\infty, 25 \mathrm{~N} / \mathrm{m}, 15 \mathrm{~N} / \mathrm{m}$, and $5 \mathrm{~N} / \mathrm{m}$. The positions of $\mathrm{Na}^{+}$and $\mathrm{Cl}^{-}$ions are labeled. Map sizes and respective color scales are identical with those of Figs. 2(a) and 2(c). The broadening and narrowing of local force fields associated with $\mathrm{Cl}^{-}$and $\mathrm{Na}^{+}$ions, respectively, as tip stiffness decreases, is clearly observable.

peculiar structure and distinct differences in interaction maps along the two directions:

(1) Lateral force values experienced by the tip apex vanish on top of individual $\mathrm{Na}^{+}$and $\mathrm{Cl}^{-}$ions, in line with previous experimental results on $\operatorname{KBr}(001) .{ }^{17}$ The reason for this finding is structural symmetry: For both crystallographic directions, the tip apex, when situated exactly on top of an ion, is exposed to the same number and type of ions on both sides, thus leading to a vanishing lateral force.

(2) The lateral force values between individual ions, however, feature contrasting characteristics for the two directions: For the [100] direction, again in accordance with experimental work performed on the ionic surface of $\operatorname{KBr}(001),{ }^{17}$ the apex is either pushed to the left or right, based on whether a $\mathrm{Cl}^{-}$ion is situated to the left (and a $\mathrm{Na}^{+}$ion to the right) or right, with the lateral force value reaching a maximum between a $\mathrm{Cl}^{-}$and a $\mathrm{Na}^{+}$ion and vanishing towards both directions [Fig. 2(b)]. In contrast, the lateral force field between individual $\mathrm{Na}^{+}$ions in the [110] direction features a more complex dual structure, in the sense that the lateral force value vanishes between the two ions and lateral forces with reverse directions are found next to this spot [Fig. 2(d)].

Having now established the qualitative and quantitative characteristics of vertical and lateral force interactions experienced by our model tip in the ideal case $\left(k_{\mathrm{x}}=\infty\right)$, the effect of lateral tip stiffness on such interactions may be studied. The procedure for simulating stiffness-affected interaction maps is as follows: Lateral forces $\left(F_{\mathrm{x}}\right)$ experienced by the tip apex at each $(x, z)$ position are calculated as in Figs. 2(b) and 2(d). Lateral relaxations undergone by the tip at each of those locations $(\Delta x)$ are then simply calculated by dividing the corresponding $F_{\mathrm{X}}$ value by lateral tip stiffness $k_{\mathrm{x}}$, representing an upper boundary of deformation. ${ }^{23}$ Finally, the $F_{\mathrm{z}}$ value at position $(x, z)$ is replaced by the $F_{\mathrm{z}}$ value at position $(x+\Delta x, z)$, taking into account the sign of $\Delta x$. Previous studies ${ }^{24}$ have found that the $k_{\mathrm{x}}$ value does not change significantly as the tip-sample interaction increases. As such, it is assumed to be constant during our simulations. While our basic approach has certain limitations, especially as the rather simplistic tip model does not include atomicscale relaxations in the apex itself (which would require more involved tip and interaction models ${ }^{8,25}$ ), it nevertheless serves our main purpose of providing a general, qualitatively accurate understanding of the effect of lateral tip stiffness on atomic-resolution force spectroscopy maps.

Figure 3 displays 2D maps of $F_{\mathrm{z}}$ for both [100] and [110] directions calculated with $k_{\mathrm{x}}$ values of 25,15 , and $5 \mathrm{~N} / \mathrm{m}$, together with the ideal $F_{\mathrm{Z}}$ maps from Fig. 2 for comparison. It is clearly evident that lateral elastic deformations undergone by the tip cause changes in the vertical interaction force maps. While repulsive force maxima situated on $\mathrm{Na}^{+}$ions become increasingly narrow with decreasing lateral stiffness, attractive force maxima on top of $\mathrm{Cl}^{-}$ions become significantly wider. When compared with the ideal case, one can see that the attractive force maxima observed on $\mathrm{Cl}^{-}$in the [100] direction at a tip-sample distance of $3 \AA$ are $\sim 10 \%$ wider at a tip stiffness of $25 \mathrm{~N} / \mathrm{m}$ and $\sim 70 \%$ wider at a tip stiffness of $5 \mathrm{~N} / \mathrm{m}$. Similar values are observed when the narrowing of the local interaction fields on $\mathrm{Na}^{+}$ions is considered for both directions. The physical mechanism behind the distortion in the spectroscopy maps can be explained with the following example: A positively charged tip apex approaching a $\mathrm{Cl}^{-}$ion from either side will be pulled toward it by a certain amount. As such, the attractive force recorded at a given $(x, z)$ position in the vicinity of the $\mathrm{Cl}^{-}$ion will increase, as the apex will now experience the force at a position $(x+\Delta x, z)$ that is actually closer to the $\mathrm{Cl}^{-}$. 
Consequently, the attractive interaction region around the $\mathrm{Cl}^{-}$ion will broaden, leading to the enlarged attractive local force fields experienced by softer tips in the vicinity of $\mathrm{Cl}^{-}$ ions. Similar arguments may be used to explain the reverse effect for local force fields associated with $\mathrm{Na}^{+}$ions. Considering that contrast changes involving the size and shape of force maxima with changing tip-sample distance are frequently observed on binary ionic crystals in force mapping experiments (most notably, in Refs. 6, 7, and 11), the importance of the presented results in terms of the effect of tip elasticity on atomic-scale interaction force mapping becomes clear.

\section{SUMMARY AND CONCLUSIONS}

Using a model tip apex and simple analytical potentials, the effect of lateral tip stiffness on atomic-resolution force spectroscopy experiments has been theoretically investigated on an ionic crystal surface. The results detailed in the previous section lead to certain conclusions:

(1) The effect of elastic, lateral deformations of the tip apex on force interaction spectra heavily depends on the lateral stiffness value of the specific tip used in the experiments. While some tips will be particularly prone to distortions, especially those that are likely contaminated by large, dangling molecules or nanoclusters from the sample surface, ${ }^{11}$ others will be able to collect spectroscopy data, which are quite similar to an ideal $\left(k_{\mathrm{x}}=\infty\right)$ scenario, assuming a high degree of structural symmetry.

(2) Due to point (i), the reproducibility of atomic-resolution force spectroscopy experiments is severely hampered. Force spectroscopy experiments performed with different tips, even if they are almost ideally symmetric, may lead to qualitatively different results based on the variability of tip stiffness values.

(3) The distortions caused by lateral tip deformations are not limited to vertical 2D interaction maps; in fact, the effects will be observable in regular NC-AFM images as a significant broadening of bright spots associated with either $\mathrm{Cl}^{-}$or $\mathrm{Na}^{+}$ions as the tip-sample distance changes. In the additional case of a structurally asymmetric tip apex, atomic-resolution images acquired at different heights may feature significantly different contrasts.

When considered along with previous studies of tip asymmetry effects on force field spectroscopy measurements, ${ }^{5}$ our results make the importance of employing structurally and chemically well-defined tips in such experiments increasingly clear. In addition to recent efforts aimed at controlling the chemistry of the tip apex, ${ }^{26}$ there is also a need to standardize the elastic properties of the tip as much as possible to augment experimental reproducibility.

\section{ACKNOWLEDGMENTS}

The authors would like to thank O. E. Dagdeviren and H. Hölscher for useful discussions.

${ }^{1}$ D. A. Bonnell et al., Rev. Mod. Phys. 84, 1343 (2012).

${ }^{2}$ C. Barth, A. S. Foster, C. R. Henry, and A. L. Shluger, Adv. Mater. 23, 477 (2011).

${ }^{3}$ J. V. Lauritsen and M. Reichling, J. Phys.: Condens. Matter 22, 263001 (2010).

${ }^{4}$ M. Z. Baykara, T. C. Schwendemann, E. I. Altman, and U. D. Schwarz, Adv. Mater. 22, 2838 (2010).

${ }^{5}$ M. Z. Baykara, O. E. Dagdeviren, T. C. Schwendemann, H. Monig, E. I. Altman, and U. D. Schwarz, Beilstein J. Nanotech. 3, 637 (2012).

${ }^{6}$ S. Fremy, S. Kawai, R. Pawlak, T. Glatzel, A. Baratoff, and E. Meyer, Nanotechnology 23, 055401 (2012).

${ }^{7}$ S. Kawai, T. Glatzel, S. Koch, A. Baratoff, and E. Meyer, Phys. Rev. B 83, 035421 (2011).

${ }^{8}$ M. A. Lantz, R. Hoffmann, A. S. Foster, A. Baratoff, H. J. Hug, H. R. Hidber, and H. J. Guntherodt, Phys. Rev. B 74, 245426 (2006).

${ }^{9}$ B. J. Albers, T. C. Schwendemann, M. Z. Baykara, N. Pilet, M. Liebmann, E. I. Altman, and U. D. Schwarz, Nanotechnology 20, 264002 (2009).

${ }^{10}$ T. R. Albrecht, P. Grutter, D. Horne, and D. Rugar, J. Appl. Phys. 69, 668 (1991).

${ }^{11}$ B. Such, T. Glatzel, S. Kawai, S. Koch, and E. Meyer, J. Vac. Sci. Technol. B 28, C4B1 (2010).

${ }^{12}$ MATLAB computing environment and programming language developed by MathWorks, Natick, MA, USA.

${ }^{13}$ M. P. Allen and D. J. Tildesley, Computer Simulation of Liquids (Oxford University Press, Oxford, 1989).

${ }^{14}$ S. Kawai, F. F. Canova, T. Glatzel, T. Hynninen, E. Meyer, and A. S. Foster, Phys. Rev. Lett. 109, 146101 (2012).

${ }^{15}$ S. Kawai, F. F. Canova, T. Glatzel, A. S. Foster, and E. Meyer, Phys. Rev. B 84, 115415 (2011).

${ }^{16}$ G. Teobaldi, K. Lammle, T. Trevethan, M. Watkins, A. Schwarz, R. Wiesendanger, and A. L. Shluger, Phys. Rev. Lett. 106, 216102 (2011).

${ }^{17}$ K. Ruschmeier, A. Schirmeisen, and R. Hoffmann, Phys. Rev. Lett. 101, 156102 (2008).

${ }^{18}$ G. H. Enevoldsen, H. P. Pinto, A. S. Foster, M. C. R. Jensen, A. Kühnle, M. Reichling, W. A. Hofer, J. V. Lauritsen, and F. Besenbacher, Phys. Rev. B 78, 045416 (2008).

${ }^{19}$ H. Holscher, U. D. Schwarz, and R. Wiesendanger, Europhys. Lett. 36, 19 (1996).

${ }^{20}$ H. Holscher, U. D. Schwarz, and R. Wiesendanger, Surf. Sci. 375, 395 (1997).

${ }^{21}$ H. Holscher, U. D. Schwarz, O. Zworner, and R. Wiesendanger, Phys. Rev. B 57, 2477 (1998).

${ }^{22}$ P. Steiner, R. Roth, E. Gnecco, T. Glatzel, A. Baratoff, and E. Meyer, Nanotechnology 20, 495701 (2009).

${ }^{23} \Delta x$ values calculated in this simple way are within $75 \%-90 \%$ of actual lateral deformation values calculated by taking into account the lowering of lateral forces in the direction of tip movement.

${ }^{24}$ A. Socoliuc, R. Bennewitz, E. Gnecco, and E. Meyer, Phys. Rev. Lett. 92, 134301 (2004).

${ }^{25}$ T. Trevethan, M. Watkins, and A. L. Shluger, Beilstein J. Nanotech. 3, 329 (2012).

${ }^{26}$ L. Gross, F. Mohn, N. Moll, P. Liljeroth, and G. Meyer, Science 325, 1110 (2009). 